Fecha de recepción: abril 2020

Fecha de aceptación: mayo 2020

Versión final: junio 2020

\section{La música como estrategia de comunicación para la resistencia de la invasión minera en la amazonia ecuatoriana}

Kléver Rolando Samaniego Pesantez ${ }^{(1)}$

Resumen: En el presente artículo se realizará un ejercicio de aproximación teórica y metodológica que permita observar a la música de los pueblos pluriculturales de la provincia de Morona Santiago de la república del Ecuador como estrategia de comunicación para la resistencia de la invasión minera planificada por el Estado nacional a través de empresas tras nacionales. Para ello se realizará una exploración, desde la comunicación, por el acontecimiento social denominado Upano Fest, evento que se desarrolla año tras años en la ciudad de Macas, con la intención de observar como, desde la música, la juventud se une para afrontar dicha intervención.

Palabras clave: Música - Estrategia de comunicación - Resistencia - Invasión minera Amazonia.

[Resúmenes en inglés y portugués en la página 201]

(1) Tiene formación académica en la Licenciatura de Ciencias de la Comunicación Social en Universidad de Cuenca-Ecuador, Máster en Gestión de La Comunicación Política y Electoral de la Universidad Autónoma de Barcelona-España y Doctor en Comunicación de la Universidad Nacional de la Plata-Argentina, ecuatoriano, cenitp@hotmail.com

\title{
Introducción
}

Los avances de la civilización han traído consigo el desarrollo industrial, que fundamenta su progreso en la explotación de los recursos naturales, especialmente en la minería. La amazonia ecuatoriana, por la amplia riqueza mineral que guarda su subsuelo, siempre ha merecido la mirada de occidente, no solo, ni únicamente como una forma de escalar niveles en el desarrollo en lo industrial y con ello en lo económico, sino también, como una forma de homogenización de lo cultural.

En el presente artículo se hace un ejercicio de aproximación para observar el evento musical Upano Fest con intenciones de mirar a su música como una estrategia de comunicación para la resistencia de la invasión minera planificada por el Estado ecuatoriano mediante empresas tras nacionales. Para ello, se explora por la dimensión comunicacional 
implementada por un grupo de jóvenes pertenecientes a la ciudad de Macas, capital de la provincia de Morona Santiago de la República del Ecuador. Observaremos como, desde su cosmovisión, defienden a la naturaleza de la implacable invasión minera de las tropas económicas que buscan la extracción de las riquezas minerales existentes en el subsuelo de la amazonia ecuatoriana.

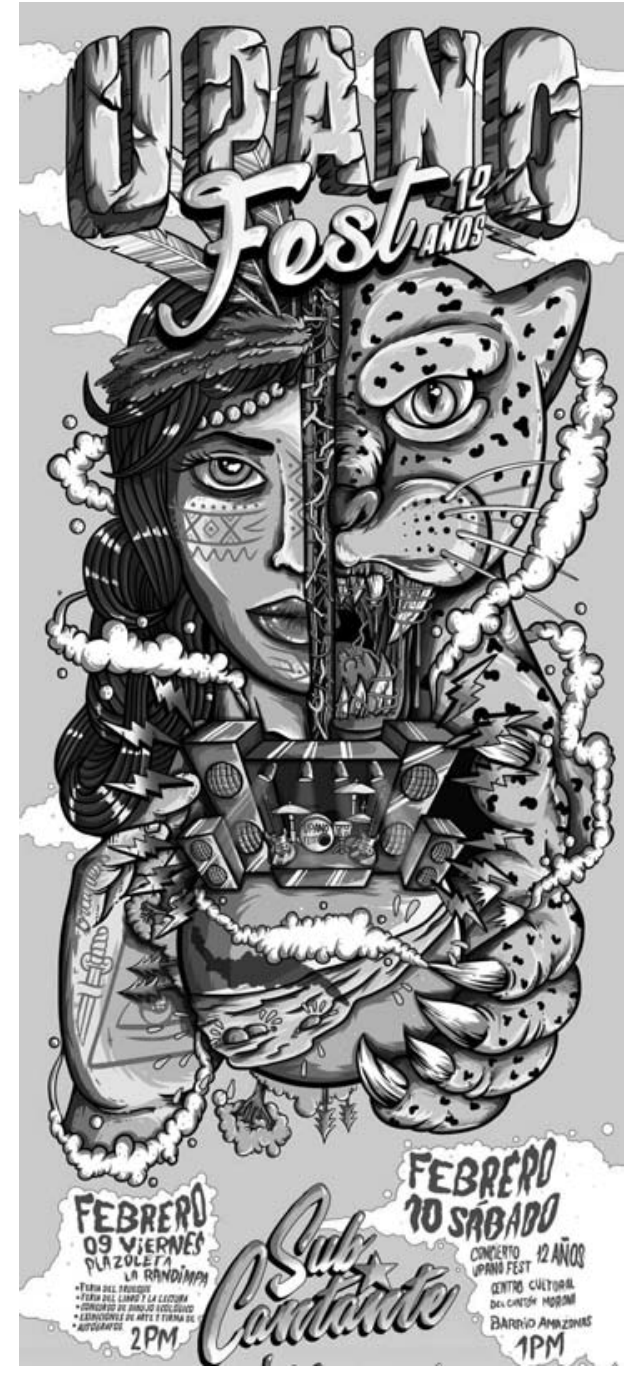

Figura 1. Afiche

promocional del Festival

Ecológico de Arte y

Música Upano Fest 12

años (Cárdenas, 2018). 


\section{Poder, resistencia y comunicación}

Este trabajo se vincula con uno de los aspectos menos explorados en los conflictos sociales, la preocupación por explicar la vida cotidiana de los pueblos dominados, con una mirada desde la ciudadanía y no desde el Estado. Este interés pasa por hacer un acercamiento al estado del arte de los conceptos de poder-resistencia-comunicación, entendiendo que las relaciones entre estado-ciudadanía son relaciones de poder, donde los dueños de las decisiones -Estado- tiene el poder de disponer la intervención con fines de explotación minera y los ciudadanos tienen el poder de resistir esa intervención. Pero estas tramas que construyen al ser humano en su condición de ser social pueden ser vistas, analizadas y estudiadas en su totalidad, como relaciones de poder. Tanto el poder como la resistencia entran en tensión en un territorio enmarcado por la comunicación.

El Estado como máximo ente de la organización social, en un determinado territorio, busca un marco normativo que le brinde estabilidad social y política. Es allí donde la mayoría de Estados han elegido los beneficios que proporciona la democracia por ser un sistema que puede otorgar esa estabilidad social -relativa-, puesto que formalmente se entiende como el poder en el pueblo. El sistema democrático consolida la vinculación entre la sociedad-Estado/Estado-sociedad, a partir de las "relaciones de poder". Esto se enmarca en las actuaciones de "quién decide, quién manda y quién obedece, quien hace" (Marván, 1999, p. 264). A partir de esto son varias las concepciones que se tienen para analizar y describir el ejercicio del poder; la instauración de la institucionalidad, las formas de ejercer los derechos, deberes y garantías desde la ciudadanía y desde un Estado, cualquiera que sea (Navarro, 2006).

El poder como concepto se empieza a desarrollar con fuerza a partir de la propuesta de Weber elaborada en: "Economía y Sociedad" (1922). Max Weber definió al poder como la "probabilidad de imponer la propia voluntad, dentro de una relación social, aun contra toda resistencia y cualquiera que sea el fundamento de esa probabilidad" (Weber, 1922-, 2002 , p. 43). Aunque consideraba la relación social como una conducta plural recíprocamente referida y orientada por tal reciprocidad, redujo conceptualmente el poder dentro de ella al resultado neto de una imposición unilateral, más allá de cualquier fundamento y, por consiguiente, negando uno o varios de los conceptos de la relación referidos a quienes padecen la imposición de la voluntad. De allí en adelante sus estudios sobre sociología de la dominación se centrarían en la comprensión de los motivos de la obediencia voluntaria, inherentes a la dominación legítima.

Dos décadas después, Ferrero (1942) configuro una noción diferente del poder, basándose en que la fuerza y la violencia voluntaria son elementos connaturales al mismo poder. En suma, los principios de legitimidad son justificaciones del poder, es decir, del derecho a mandar. Entre todas las desigualdades humanas, ninguna tiene tanta necesidad de justificarse ante la razón como la desigualdad establecida por el poder.

Por otra parte, Múnera (2005) acota a esta discusión mencionando que el poder es "una acción colectiva concertada que le otorga legitimidad al apoyo activo o a la obediencia voluntaria dentro de una comunidad o asociación" (p. 36). Propone mirar al poder en una acción de consenso desplazando a la fuerza del consenso. Para realizar su propuesta, el autor parte de Marx (1922), quien no lo aborda de forma directa, pero se observan 
algunos elementos que facilitan inferirlo. Para Marx (1922), el poder se sitúa en el marco de las relaciones de producción y siguiendo esta línea, para Múnera (2005), es un dominio ejercido por la clase social determinada sobre el proletariado.

Dentro de la misma línea, Habermas (2000) realiza un análisis de la concepción del poder de Hannah Arendt (1950), donde diferencia entre poder comunicativo -como aquello que surge cuando los hombres se reúnen para actuar y dialogar en concierto-, poder administrativo -el ejercicio mismo del poder dentro del sistema o jerarquía de cargos establecidos por las leyes, que implicaría una lucha por las posiciones que facultan su uso-, y el poder social -la fáctica capacidad de imponerse que tienen los intereses privilegiados-.

Foucault $(1986,1975,1976,2001,1991)$ plantea entender el poder desde una perspectiva relacional, lo que le permite constituirse en uno de los autores más relevantes frente al análisis del tema del poder, en particular porque no lo asume desde una mirada jerárquica, sino que analiza las estructuras de poder.

El poder no es considerado como un objeto que el individuo cede al soberano, el poder es una relación de fuerzas, una situación estratégica en una sociedad en un momento determinado. Foucault (1976) reflexiona que el poder se presenta a través de toda la sociedad en el marco de las relaciones sociales, por lo que resulta apenas natural que los procesos comunicativos, comprendidos como el escenario por excelencia, en el cual se generan las relaciones sociales, estén en el epicentro de las relaciones de poder. Para Foucault (2001, $2005,2008)$ y Bourdieu $(1999,2000)$, la comunicación juega un rol esencial en las relaciones sociales siendo capaz de producir efectos de poder (Vázquez, 2002).

Los aportes de Foucault $(2001,2005,2008)$ y Bourdieu $(1999,2000)$ permiten pensar el poder como relaciones de comunicación (Saintout, 2010), no desde su dimensión representativa sino constitutiva de legitimidad, productiva de conocimiento que legitima la dominación. Esta nueva perspectiva abrió un campo de indagaciones para una teoría crítica de la cultura desde la comunicación.

El poder no es considerado como un objeto que el individuo cede al soberano, el poder es una relación de fuerzas, una situación estratégica en una sociedad en un momento determinado (Samaniego, 2018). Foucault (1976) reflexiona que el poder se presenta a través de toda la sociedad en el marco de las relaciones sociales, por lo que resulta apenas natural que los procesos comunicativos, comprendidos como el escenario por excelencia, en el cual se generan las relaciones sociales, estén en el epicentro de las relaciones de poder. Para Foucault $(2001,2005,2008)$ y Bourdieu $(1999,2000)$, la comunicación juega un rol esencial en las relaciones sociales siendo capaz de producir efectos de poder (Vázquez, 2002). Desde el concepto de poder realizado por Foucault (1975) buscaremos nuestro objetivo;

una acción sobre las acciones de los otros el poder no se posee, sino que se ejerce, y no es el privilegio adquirido de la clase dominante, sino el efecto de conjunto de sus posiciones estratégicas. No se aplica pura y simplemente, como una obligación o una prohibición a aquellos que no lo poseen; los invade, pasa por ellos y a través de ellos (pp. 33-35).

Por su parte el concepto de resistencia, originalmente proviene de la polemología -estudio científico de la guerra como fenómeno social-, y se entendía como una acción militar 
frente a una invasión, por ejemplo, la resistencia de los indios de la Sierra Gorda a la invasión norteamericana o la de Juárez ante la intervención francesa o la de los Azhuar ante la conquista española. Posteriormente, y por analogía, el concepto se extendió y se aplicó también a la lucha de los campesinos en defensa de sus tierras contra la hacienda. En el caso de investigadores de habla inglesa la resistencia se entendía como un evento violento en el que participaba una comunidad agredida en un momento particular de su historia. A partir de los años 80 el concepto de resistencia se amplió a discursos y gestos-implícita o explícitamente impugnativos- de la vida cotidiana u ordinaria de los subalternos, interpretados como una infrapolítica de los desvalidos (Scott, 1985, 2000) o también como el "arsenal de los pobres" para su defensa frente a todo tipo de poder. La publicación del libro del antropólogo norteamericano James C. Scott (1985), Weapons of the Weak: Every Forms of Peasant Resistance, trajo agua fresca al molino de los estudiosos de los movimientos campesinos.

Si analizamos algunos de los postulados encontramos a Randle (1998), Thoreau (1995) Sharp (1973), Gandhi (2010) donde se puede ver que existen grandes avances en el desarrollo de sus definiciones; aunque el fenómeno de desobedecer o de resistir frente al Gobierno es más algo antiguo. Thoreau (1995) al partir de un caso como el norteamericano en el que se puede hablar de una resistencia contra un gobierno "civil", también tiene en cuenta una visión pragmática y moral de la resistencia, por lo que ha sido estudiado por pensadores como Sharp (1973), que se centran en lo pragmático, hasta personalidades como Gandhi (2010), que, sin perder de vista los métodos, les da mayor importancia a los valores como desobedecer a la injusticia proveniente de un Gobierno.

$\mathrm{Al}$ leer a los autores descritos se puede identificar que sus posturas derivan del postulado de Michel Foucault (1976) según el cual "donde hay poder, hay resistencia", pero a veces ésta aparece como una resistencia estructural según el principio de Arquímedes, es decir, mecánica, en lugar de ser el resultado de una acción política consciente y elaborada, aunque ninguno de los autores de habla inglesa descritos se interroga sobre el origen de la resistencia, sin embargo, el desenlace parece claro; la acumulación de resistencias constituye un gran arrecife donde puede encallar la nave del Estado, considerando que el poder provoca, por su solo ejercicio, una contra-respuesta entre los dominados, sin intervención explícita de alguna organización política (Héau, 2007).

En las corrientes latinoamericanas, se puede ver que la resistencia es uno de los conceptos y de las ideas más amplias y genéricas. Tienen relación con un conflicto que nace, con la presencia y el dominio de los imperios, principalmente europeos. En este sentido, el conflicto emerge por el vínculo de dominio y opresión que se crea hacia los pueblos autóctonos o indígenas. Para deshacer estos vínculos de dominio, la lucha está acompañada tanto de combates armados como de no armados. López Martínez (2013), menciona que existen varios ejemplos a lo largo de la historia en los que se evidencia una resistencia no armada frente a la dominación colonial.

Para autores como Randle (1998), Arendt (2005a, 2005b), Habermas (2000) Hernández (2004), Ballesteros (2005) y González Piñeros (2006), todos concuerdan que la resistencia son acciones ciudadanas se debe denominar como resistencia civil porque se encuentra en un marco más amplio, como los métodos de acción no violenta. Si se toman algunos elementos de los que se tienen en cuenta para definir la resistencia civil, podemos ver que 
se relacionan estrechamente con los preceptos y las ideas impulsados desde la teoría de la no violencia.

Si se entra en detalle, el concepto de desobediencia civil tiene una connotación específica y es la de encontrarse en contraposición con una norma o una ley establecida, que se considera injusta o nociva (Ballesteros, 2005). Se diferencia del concepto de resistencia civil en la medida en que puede ser entendido como un método dentro de ella, es decir, la desobediencia civil puede ser una forma de acción dentro de la misma resistencia civil. La desobediencia civil, al igual que la resistencia civil, hacen parte de la estructura más amplia, como es la del pensamiento no violento. Otro tema que es importante revisar es el concepto de resistencia de las comunidades indígenas quienes piensan en la armonía y el bienestar de sus comunidades desde todo lo que engloba la idea de Sumak Kawsay -el buen vivir- hacen que los medios y los fines tengan gran compatibilidad, asunto que facilita la contrastación de la teoría no violenta con la práctica propia y específica (Martínez, 2016). Es sobre este concepto que continuaremos en nuestra investigación, claro apoyados en la Ciencia de la Comunicación Estratégica que menciona que el diálogo de saberes situados es otro eje articulador de su especificidad. Esto implica partir del reconocimiento de que fuera del ámbito académico se generan también conocimientos válidos y socialmente necesarios, con los que los comunicadores deben poner en común para abordar lo que con frecuencia no permite ver la mirada disciplinaria, fragmentada y analítica de las ciencias sociales tradicionales.

Situarse en y desde la perspectiva de los estudios en comunicación implica desplazarse del examen teórico de los medios y la determinante tecnológica hacia los contextos, mediaciones y las prácticas culturales (Aguerre, 2017). Esto implica examinar los fenómenos sociales no desde el lugar instrumental sino relacional (Uranga, 2008), con una mirada que enfoque a la naturaleza como sujeto portador de derechos y a la comunicación como un derecho fundamental y constituyente del hombre y de lo que lo rodea.

Hablar de comunicación es reconocer cómo los individuos se apropian del medio que los rodea y de qué manera internalizan la naturaleza en la cultura. La naturaleza es un factor de derecho y desarrollo humano, es un todo, en donde todo vive en armonía entre todos sus componentes, incluido el humano.

El derecho de la naturaleza lo debe ganar el humano, pero solo lo gana si revierte la tendencia a la fragmentación occidental de los componentes de los entornos con fines mercantiles, y recupera la visión de la totalidad, holística, compleja. Sandra Massoni (2014) menciona que "es bueno recordar que nada en el ser humano ni en la naturaleza es un fragmento. En cualquier terreno que transitemos es preciso no perder de vista el horizonte de la totalidad" (p. 3).

La misión sería, entonces, recuperar la cosmovisión de los pueblos ancestrales latinomamericanos, que miran la totalidad de sus contextos, descolonizando las relaciones en el propio territorio. 


\section{La música como estrategia}

La música es una manifestación artística, es un medio de expresión de ideas, de emociones, de vivencias de una sociedad. El arte musical combina sus elementos propios de tal forma que acerca al oyente a la cultura del pueblo que la ha producido y a las emociones humanas.

La música desde la historia ha tenido diferentes direcciones, el espacio mas conocido de es su utilización como estrategia se encuentra en el espacio educativo, ver entre otros: Avillar (2015), Sánchez (2004), Reyes (2012), Videz (2014). Sin embrago, para el presente estudio revisaremos únicamente la utilización de la música como estrategia de comunicación para guerra y de paz: la música como instrumento de paz podemos encontrarlos en Alerta, 2005; Siankope y Villa, 2004; Sanfeilu y Carieta, 2005 y la música como instrumento de guerra en Pérez, 2014, entre otros.

La importancia de la música en los conflictos bélicos era una cuestión que se había considerado desde la edad media, pero en el trascurso del siglo XIX y XX sus nuevos planteamientos antropológicos, permitieron observar detalladamente la influencia que ejercía esta expresión artística sobre los soldados. Estos estudios culturales permiten entender que la manifestación musical debía ser imprescindible en las tareas propagandísticas y en la consolidación del grupo de combatientes (Pérez, 2014), esta forma de uso de la música fue implementado por todos los países latinoamericanos, mediante la implementación de los Himnos Nacionales, mismos que hasta hoy son considerados como uno de los tres elementos simbólicos de las nacionalidades. En este sentido la estrategia de comunicación ha sido utilizada desde los enfoques del tercer paradigma estratégico, que se fundamenta en el "conflicto y soportada por una visión racionalista, dialéctica y antagónica" (Massoni y Pérez, 2009, p. 15).

Massoni y Pérez (2009) proponen un desplazamiento en la forma de mirar y aplicar la estrategia, este cambio va a la teoría de la ciencia de la articulación social más que a la ciencia del conflicto.

Desde una mirada más relacional se ha abordado a la música como una estrategia de paz, desde donde se reconoce a la música como un instrumento de lucha no violenta, para la construcción de la paz. La constitución de la música para la paz permite cambiar de perspectiva como un proceso educativo, dinámico, continuo y permanente, fundamentado en los conceptos de paz y en la perspectiva creativa del conflicto y que a través de la creación de enfoques socio afectivos y problematizantes se desarrolla un nuevo tipo de cultura, la cultura de la paz que ayude a develar críticamente la realidad para poder situarse sobre ella y poder actuar en consecuencia (Jares, 1999, p. 124).

\section{Antecedentes}

En las décadas recientes se verifica un aumento acelerado de los flujos metabólicos o biofísicos (de materiales y de energía), y consecuentemente de la explotación del trabajo aunado a un proceso estructural de despojo de los bienes comunes. El resultado es una 
correlación entre la dinámica del aumento de flujos biofísicos y la de acumulación de capital. De ahí que se estime que entre 1900 y 2000, cuando la población creció cuatro veces, el consumo de materiales y energía haya aumentado en promedio hasta diez veces: el incremento del consumo de biomasa se estima en 3,5 veces, el de energía en 12 veces, el de metales en 19 veces y el de materiales de construcción, sobre todo cemento, unas 34 veces (Krausmann, 2009). Al cierre del siglo XX la extracción de recursos naturales fue de 48,5 mil millones de toneladas (más de una tercera parte biomasa, 21\% combustibles fósiles y $10 \%$ minerales), registrándose un consumo global per cápita de 8.1 toneladas al año con diferencias per cápita de más de un orden de magnitud (Steinberg, Krausmann y Eisenmenger, 2010). Para 2010 las estimaciones rondan las 60 mil toneladas de materiales al año y unos 500 mil petajoules de energía primaria (Weisz y Steinberger, 2010). El 10\% de la población mundial acaparaba el $40 \%$ de la energía y el 27\% de los materiales (Ibid). Estas estadísticas, sumadas a la promulgación de la industria están generando un serio calentamiento global que están generando problemas con el hogar en donde vivimos, es decir, el planeta Tierra. Esta situación ha sido advertida por científicos de muchos países como el gran problema a resolver. Al contrario de lo esperado, luego de las grandes encuentros mundiales sobre cambio climático y medio ambiente, como la de Brasil (1992), Río de Janeiro; Japón (1997), Protocolo de Kioto; Sudáfrica (2002), Johannesburgo; México (2010), Cancún; y Brasil (2012), Río+20; principalmente, que han dejado al descubierto que los países del primer mundo industrial son, paradójicamente, los menos decididos a disminuir las emisiones de gases tóxicos que producen el efecto invernadero y lesiones para la atmósfera terrestre. Este comentario se puede aseverar luego de la negativa a disminuir la producción y la implementación de su gran máquina industrial ${ }^{1}$. Los países considerados como del primer mundo no han firmado varios tratados, demostrando con ello que más les importa las ganancias del capital que el futuro del planeta Tierra y sus diversas formas de vida.

Bajo ese marco, la república del Ecuador, ha realizado pasos agigantados con relación a la consolidación de un marco normativo que permita mirar a la naturaleza como un sujeto de derechos. En ese sentido, el primer aporte, se registra en la constitución de 1978, en el artículo 22, numeral 2, en donde se establecía que los ecuatorianos teníamos:

El derecho a vivir en un medio ambiente libre de contaminación. Es deber del Estado velar para que este derecho no sea afectado y tutelar la preservación de la naturaleza. La Ley establecerá las restricciones al ejercicio de determinados derechos o libertades, para proteger al medio ambiente (CN, 1978, art 22, n 2).

Los derechos de la naturaleza constituyen una hecatombe para la tradición jurídica francesa romanista (...) Para la abolición de la esclavitud, se requería que se reconozca "el derecho de tener derechos" y se requería también un esfuerzo político para cambiar a las leyes que negaban esos derechos. Para liberar a la Naturaleza de esta condición de sujetos sin derechos o de simple objeto de propiedad, es necesario un esfuerzo político que reconozca que la naturaleza es sujeto de derechos (Trujillo, 2010, pp. 121-123). 
Para dotarle de derechos a la Naturaleza, en Ecuador, significó alentar políticamente a un despliegue del sentido y noción de naturaleza. Es decir, pasar de objeto a sujeto. Este paso dado en la constitución aprobada en el año 2008, fue el resultado de un proceso centenario de ampliación de los sujetos del derecho.

En Ecuador, la Naturaleza o Pachamama, es sujeto de derechos y por tanto, se debe respetar en forma integral su existencia; así como el mantenimiento y regeneración de sus ciclos vitales. Es responsabilidad del Estado ecuatoriano, incentivar a las personas naturales o jurídicas y/o los colectivos, protejan a los derechos de la Naturaleza; así como el promover el respeto de todos los elementos que forman un ecosistema (Paredes, 2014).

Sin embargo, el mismo Gobierno que inscribió los derechos de la naturaleza, en la constitución del año 2008, crea La Empresa Nacional Minera del Ecuador ENAMI EP, en el año 2010, como parte de la política para la gobernanza responsable y sostenible de los recursos naturales. Esta empresa pública, en el año 2016 aprueba el portafolio de proyectos para la explotación minera en cinco proyectos entre uno de ellos "Panantza-San Carlos".

Este proyecto de minería en mención, se encuentra situados en de la parroquia San Carlos de Panantza, en el cantón San Juan Bosco, Morona Santiago.

\section{Objeto de estudio}

El Colectivo Social Upano Fest, es un movimiento social que tiene como icono el rio Upano, que nace en el volcán Sangay, cruza principalmente por las ciudades de Macas y Sucúa perteneciente a la provincia de Morona Santiago en la zona amazónica de la Republica del Ecuador. En todo su trayecto alberga fauna silvestre.

Por la incidencia negativa que provoca la pequeña minería que trabajaba en y cerca del rio Upano, surge un colectivo social con el objetivo de parar estas malas prácticas, su primera estrategia de comunicación fue la denominada Randimpa Juvenil de eco-limpieza y de reforestación sobre el río Upano, consiste en realizar actividades de beneficio público con la participación de los comuneros, jóvenes activistas ambientales, que busquen la conservación de la biodiversidad existente en el lugar o que resulten afectados o beneficiados de esa actividad. Sin embargo, el Estado y la sociedad civil del resto del país no tienen oídos para este grupo de jóvenes.

Luego de los fallidos intentos por ser escuchados emerge una visión compleja sobre la utilización de la música para llamar la atención de medios de comunicación y así la sociedad ecuatoriana en su conjunto.

El movimiento social Upano Fest crea un festival internacional de música que lleva el mismo nombre del movimiento social con la intención de que artistas de otros países y locales tenga la oportunidad de cantar en un lugar paradisiaco rodeado de naturaleza nativa, en la selva de la Amazonia.

Este evento no se reduce a un concierto, que también es importante, sino a la generación de una estrategia que busca en la complejidad, la transformación social que va desde diferentes tipos de público objetivo entre otros, estudiantes de escuelas, colegios y universi- 
dades, así como comerciantes amas de casa, entre otros. El público objetivo y la estrategia para visitar sus lugares de convergencia.

Si bien este evento es financiado por el Estado ecuatoriano, los organizadores buscan mantener las libertades de pensamiento cultural, es por esa razón que jamás se permite el ingreso de temas políticos partidistas en mencionado evento.

El festival ecológico de Arte y música Upano Fest es un encuentro de músicos independientes ecuatorianos, realizado en la ciudad de Macas, Provincia de Morona Santiago, desde el año 2007. Este festival reúne a gran parte de artistas con su música, cultura e ideología, unidos comparten el mensaje de conservación y cuidado del medio ambiente en la selva ecuatoriana y a la vez, ser un espacio en donde la juventud pueda expresarse libremente de forma sana. El festival se celebra en temporada de Carnaval, sirviendo además como un encuentro turístico de gran importancia realizado para todo tipo de público, enfocado en los jóvenes, que gustan de eventos donde existen diferentes actividades relacionadas con labor comunitaria, entre otros esta la "Randimpa Juvenil, Eco Limpieza, Danza y folclore de la Amazonia, Feria del Trueque, Feria del disco independiente, concurso de dibujo y pintura, es un espacio abierto para compartir pensamientos, escuchar nuevas propuestas musicales independientes de Ecuador y Latinoamérica y defender a la naturaleza de la invasión minera capitalista" mencionó Daniel Noguera uno de los principales dirigentes del colectivo (Noguera, D, comunicación personal, 11 de noviembre 2017).

\section{La comunicación como perspectiva de abordaje}

El propósito de este proyecto de investigación es reflexionar sobre el aporte que realiza la comunicación a la defensa de los tesoros culturales, minerales, naturales y conocimientos míticos -derecho a la naturaleza-, que cubre la corteza terrestre en la amazonia ecuatoriana. Para ello, nos situamos en la línea de los investigadores ${ }^{2}$ que trabajan en la perspectiva de comunicación entendida como un derecho fundamental para la vigencia plena de los derechos humanos. Este marco teórico de la comunicación es subyacente al ejercicio pleno de los derechos humanos. Derechos que son interdependientes y que no pueden ser disociados. Los derechos humanos abarcan la libertad, la participación, la solidaridad, el acceso, la inclusión, la equidad, y la justicia.

Todos los derechos humanos representan la posibilidad de elegir y de utilizar nuestro criterio como seres humanos. Son derechos que debemos ejercer de manera cotidiana. La comunicación se presenta como el derecho articulador de otros derechos, es un proceso humano de relación, que implica no solamente intercambio de información, sino puesta en común de conocimientos y reconocimientos de las diferencias. Descrito por Gumucio

El derecho a la comunicación articula y engloba al conjunto de otros derechos relativos, como son el acceso a la información, la libertad de opinión, la liber- 
tad de expresión, la libertad de difusión. (...) un derecho de todas y todos, que implica el acceso y la participación en procesos individuales y colectivos de construcción de conocimiento (Gumucio, 2012, p. 1).

Para Uranga (2016 -b-) "la comunicación es un derecho fundamental y una condición esencial para la vigencia plena de los derechos humanos" (p. 5). Bajo esa perspectiva entendemos a la comunicación como un derecho humano fundamental (Uranga, 2016 -a), un derecho habilitante de otros derechos (Massoni, 2017) entre ellos el derecho a la naturaleza. Por lo tanto, una puerta de entrada al análisis del derecho a la naturaleza desde la comunicación.

Bajo ese marco pretendemos realizar un aporte a la re-significación del aporte de la comunicación a la protección del derecho a la naturaleza. Dentro de ese marco se encuentra la Comunicación Estratégica Enactiva (Massoni, 2003) como la ciencia de la comunicación y no solo como una herramienta de abordaje de otras ciencias.

\section{Metodología de la investigación desde la comunicación}

La metodología de investigación que habla este apartado se despliega de la teoría de la Comunicación Estratégica Enactiva (Massoni, 2003), la cual se sitúa en el cruce de los nuevos paradigmas y las corrientes latinoamericanas que realiza Sandra Massoni (2000, 2003, 2005a, 2005b, 2006, 2007a, 2007b, 2007c y 2009).

Este modelo propone pensar la estrategia desde una perspectiva inclusiva, conciliadora y articuladora. Busca reconocer y reconocerse en los saberes situados. Este desplazamiento teórico toma distancia de la ciencia que mira a la estrategia como una herramienta de guerra y de conflicto.

La Comunicación Enactiva es una metodología de examen comunicacional que posee herramientas, técnicas y metodologías propias de los comunicadores, en tanto proyecto de Investigación Enactiva en Comunicación (IEC).

La Comunicación Enactiva, propone a las estrategias de comunicación como dispositivos micro/macro social conversacional, especializados en el análisis y la operación de lo comunicacional (Massoni, 2013 -a-).

La Comunicación Enactiva habilita, inquiere, busca la enacción de otras modalidades de encuentro a partir de la constitución de un cambio social conversacional que se ofrece como un valor agregado a los modelos de investigación tradicional.

En el recorte comunicacional de la Comunicación Enactiva es posible entender a la comunicación desde sus múltiples dimensiones como un fenómeno complejo, situacional, histórico y fluido (Massoni, 2003). Alejándose de los enfoques sociológicos y semiológicos clásicos que estudian a lo comunicativo en tanto significaciones transmitidas. Este nuevo enfoque estudia lo comunicacional en tanto acciones y sentidos emergentes que no se desentienden de los acoplamientos dinámicos y evolutivos de la realidad y los sujetos. Define a la comunicación como un encuentro sociocultural, en tanto espacio y momento relacionante de la diversidad macrosocial y propone a las mediaciones, a las marcas de 
racionalidad comunicacional y a las matrices socioculturales como técnicas de análisis metodológicos propios de comunicadores sociales.

La Comunicación Enactiva es una consecuencia de los trabajos científicos realizados por Sandra Massoni (1990, 2011, 2013, 2015 y 2016), que propone a la estrategia de comunicación como un dispositivo doble de inteligibilidad y de interpelación que busca, a partir de indagación, hacer emerger nuevas realidades (Massoni, 2009), propone además siete pasos para su implementación: la definición de la Versión Técnica Comunicacional, Análisis y prescripciones mediante marcas de racionalidad comunicacional, Reconocimiento y jerarquización de actores vinculados a cada componente del problema comunicacional, Caracterización de matrices socioculturales en torno al problema, Árbol de soluciones, Investigación de campo y Diseño del plan operativo oficial de la estrategia comunicacional

\section{La comunicación enactiva aplicada}

En los apartados anteriores se ha dado cuenta que la Comunicación Enactiva no es vista como transferencia de conocimiento, como un paquete a ser transmitida, no se reduce al análisis de lo comunicativo, sino que se analiza desde un enfoque multiparadigmático y transdiciplinar que concibe a la actuación de la comunicación, también, desde lo fractal y lo fluido.

Pensar a la comunicación desde el nuevo enfoque implica un doble esfuerzo; por un lado, una batería de conceptos y metodologías que habiliten nuevas formas de interpelar las realidades y por otro, un espacio de reflexión sobre el aporte y el lugar del investigador comunicador en los procesos de cambio (Massoni, 2015, p 13).

Desde esa perspectiva la Comunicación Enactiva es una exploración de la investigación comunicacional, un espacio de desarrollo creativo formulado desde la estrategia de comunicación que busca como fin ultimo las transformaciones sociales en una situación dada. El aporte de los comunicadores estratégicos se diferencia de otros investigadores sociales en que su objeto de estudio es el cambio social conversacional.

Las estrategias de comunicación son dispositivos de diseño, en tanto modalidad de investigación enactiva centrada en acompañar procesos de cambio social conversacional operando en el vínculo micro-macro social de generación de conocimiento para la innovación (Massoni 1990, 2011).

En este caso de investigación se aplicó los siete pasos de un proyecto típico de Investigación Enactiva en Comunicación, sin embrago, por cuestiones de espacio hablaremos únicamente de la matriz aplicada y sus resultados obtenidos. 


\section{Resultados y conclusiones de la implementación de la estrategia de comunicación en el espacio musical Upano Fest}

La estrategia de comunicación como dispositivo doble fue implementada en un grupo de jóvenes de la ciudad de Macas, provincia de Morona Santiago perteneciente a la republica del Ecuador.

Para ello, se realizó el desplegamos los 7 pasos de Investigación Enactiva en Comunicación, pero con diferente nivel de profundidad, es decir, que en la presente investigación se indagó lo suficiente para determinar el aporte de la comunicación para la resistencia de la invasión minera en la provincia de Morona Santiago, ubicada en la amazonia ecuatoriana. El primer dispositivo implementado fue la matriz denominada Versión Técnica Comunicacional (VTC), con la que se logró generar un dialogo de saberes. La aplicación de este formulario de realizó en un taller multiparadigmatico que contó con la presencia del grupo de jóvenes autodenominados colectivo social "Upano Fest" en la cual se visualizó que el principal problema de comunicación es la falta de promoción del evento a nivel nacional e internacional. De los resultados de esa matriz se elaboró las técnicas Marcas de Racionalidad Comunicacional (MRC), reconocimiento y jerarquización de actores vinculados al problema, caracterización de las Matrices Socicoculturales que se desprendía de la Versión Técnica Comunicacional, la construcción de las Matrices Socioculturales, implementación del árbol de soluciones y el planteamiento del plan operativo de la estrategia de comunicación inicial y con ello un diagnostico y un camino a seguir para que se logre los objetivos planteados.

\section{Conclusiones}

A través de la observación, registro y análisis (Carballeda, 2008) de las estrategias de comunicación del Colectivo Social Upano Fets, se demuestra que consiguieron poner en diálogo -la importancia de mantener, conservar y preservar el medio ambiente- a los demás actores que son parte del trama relacional de la invasión de la minería en la amazonia ecuatoriana. El colectivo social mediante y a través de la música logró, en primer lugar, generar un proceso social participativo que implicó la consideración de lo público, lo político. En segundo lugar, posibilitó constituir, a este espacio, como un entramado de discursos y un terreno de disputa simbólica en donde la comunicación emerge como un momento y espacio relacionante del encuentro sociocultural conversacional en las alteridades (Massoni, 2010), que permite la resistencia social. Es decir, un este espacio de encuentro sociocultural, no es un espacio común, es un espacio que produce cambios, es un espacio que enactúa, allí su estatus de estratégica.

Creemos que con esta serie de argumentaciones y demostraciones queda claro que el potencial de la música en la resistencia social es importante y decisivo, -hasta el momento- a partir del caso expuesto.

La Comunicación Estratégica Enactiva de la que hablamos, subraya los reduccionismos de las semióticas dominantes en el siglo XX en torno al que habla, destacando su lógica 
matemática centrada en las características de la razón económica. Alejándose de la palabra vacía, desocupada de comunicación. La Comunicación Estratégica Enactiva que parte de un espacio y momento relacionante en la diversidad sociocultural, se sustenta en una metaperspectiva específica de Morín, Capra, Nicolescu y otros expertos en transdisciplinariedad y pensamiento complejo, plantean como destino y desafío de la nueva ciencia y de la nueva profesión (Massoni, 2012).

Alrededor de la música se estructuran formas sociales de expresión y circunstancias culturales de colectivos que crean la noción de resistencia, sin la necesidad de tomar las armas sino más bien como una forma de manifestación de sentidos que emergen opuestos con las decisiones unilaterales del Estado, mismo que busca únicamente un interés económico, sin importar la cosmovisión ancestral de los dueños de los territorios, los pueblos aborígenes.

La comunicación, desde los nuevos paradigmas, se presenta como un derecho articulador de otros derechos, un derecho que permite mirar la totalidad del problema y no de una forma fragmentada o lineal. Hablamos de la comunicación como un derecho constituyente del hombre y lo que le rodea, un derecho que permite cuidar, defender y conservar el derecho a la naturaleza.

La complejidad encontrada en esta investigación está en que previo al evento se desarrollan reuniones con los músicos para definir sobre su perspectiva de participación (No a la Actividad Minera en la zona del Amazonas) y las reuniones van focalizadas al desarrollo de canciones y discursos utilizados antes durante y después de las participaciones de los grupos. Además, la estrategia es complementada las charles en las aulas de centros educativos tanto públicos y privados de toda la ciudad de Macas, espacio en donde se habla de forma abierta sobre la concientización de la importancia de mantener el medio ambiente los rodea. Las Marcas de Racionalidad Comunicacional del colectivo social se encuentran centralizadas en la dimensión interaccional y en la dimensión sociocultural, que se manifiestan en la música como eje central de la estrategia de resistencia.

Su campo de acción lo constituye el trabajo comunitario a través de presentaciones en escuelas y colegios del sector, y, esporádicamente, en colegios ubicados fuera de la localidad. Las formas de resistencia cotidianas anticipan, preparan y acompañan al trabajo político, pero por sí solas no lo reemplazan. Para saltar a la escala política desde lo ciudadano, necesitan de una mediación, un territorio, que las potencien para confrontar eficazmente al poder dominante, que en este caso es la comunicación como encuentro.

El arte no informa, únicamente, se escapa de las lógicas anatomopolíticas de las sociedades de control sino transforma en un espacio que enactua. Por ello supone una disfunción, una molestia, una incomodidad, un escape al paradigma de la información que en tanto informa ordena, imponente y acomoda. El arte es un espacio propiciado para la transformación social, un espacio donde enactuan los sentidos emergentes de la realidad intersubjetiva de los sujetos. Puede penetrar y corroer la lógica por la cual los individuos se convierten en dividuos y las masas en bancos de datos que se compran y venden. El arte crea afectos y preceptos y huye del orden, da lugar a vacuolas de no información que desarmonizan y desquician la circulación de los mensajes de orden.

La Comunicación Estratégica Enactiva no busca, únicamente, acompañar a los actores en la resistencia, va mas allá acompaña a las matrices socioculturales a persistir en su 
transformación social, en palabras de Boaventura de Sousa Santos (2019) "la resistencia esta fragmentada" y en este caso se debe propiciar su enacción porque cuando lo comunicacional se vincula con lo poblado genera crecimiento, cuando se organiza entorno a la tolerancia produce resistencia y la resistencia tiene un limite que estalla cuando se lo sobrepasa (Massoni, 2019).

\section{Notas}

1. Para mayor información consultar en: http://www.dspace.uce.edu.ec/bitstream/25000/ 3835/1/T-UCE-0013-Ab-171.pdf

2. Entre otros: José Carrera (2007); Alfonso Gumucio (2012); Sandra Massoni (2008, 2010, 2016); Washington Uranga (2007, 2008, 2016-a-); Natalia Zapata (2014).

\section{Referencias Bibliográficas}

Arendt, H. (2005a). ¿Qué es la política? Buenos Aires, Argentina. Ed. Paidós.

Arendt, H. (2005b). Sobre la violencia. Madrid, España. Ed. Alianza.

Ballesteros Peluffo, G. L. (2005). Aspectos teóricos e históricos de la desobediencia civil.

Bhabha, H. K. (1994). El lugar de la cultura. Buenos Aires, Argentina. Ed. Manantial.

Bravo, A. y Bruzzone, A. M. (1995). In guerra senza armi: storie di donne 1940-1945. Roma. Ed Italian.

Laterza Cante, F. (2005). Acción política noviolenta, una opción para Colombia. Universidad del Rosario.

La Boétie, É. (1986). Discurso de la servidumbre voluntaria o el contra uno. Madrid, España. Ed Tecnos.

Gandhi, M. (2004). Escritos esenciales. Santander: Sal Terrae.

Gandhi, M. (2010). Política de la noviolencia. Madrid, España. Ed. Catarata.

Godwin, W. (1793). An enquiry concerning political justice and its influence on general virtue and happiness. Londres, Reino Unido. Ed. GGJ and J. Robinson.

González Piñeros, N. C. (2006). Resistencia indígena: alternativa en medio del conflicto colombiano. Cali, Colombia: Pontificia Universidad Javeriana.

Gumucio, A. (2012). "El derecho a la comunicación: articulador de los derechos humanos". Revista Razón y Palabra. N. 80. Pp. 1-31.

Habermas, J. (2000). La desobediencia civil. Piedra de toque del Estado democrático de derecho y derecho y violencia. Un trauma alemán. Barcelona, España. Ed. Península.

Hernández Delgado, E. (2004). Resistencia civil artesana de paz: experiencias indígenas, afrodescendientes y campesinas. Bogotá, Colombia. Pontificia Universidad Javeriana.

Massoni, S. (2016a). Avatares del comunicador complejo y fluido. Quito, Ecuador. Ediciones Ciespal. 
Massoni, S. (2016b). "Investigación enactiva en comunicación. La participación ciudadana más allá del control en proyectos de comunicación estratégica ambiental”. En J. Astudillo y T. Villasante (Comp). Participación ciudadana con metodologías alternativas desde el sur. Cuenca, Ecuador. Ediciones Adya-Yala.

Massoni, S. (2016c). "La participación ciudadana es un componente del encuentro sociocultural conversacional”. (K. Samaniego, Entrevistador)

Massoni, S. (2015). "Comunicación estratégica: Matrices de datos en la Investigación enactiva”. En Revista Latinoamericana de Ciencias de la Comunicación. Año X No18,ALAIC,enero-junio 2013.

Massoni, S. M. (2014). "Plataforma online para el registro y operación de estrategias comunicacionales". En Revista Especializada en Periodismo y Comunicación Vol. 1, No 41, Questión, marzo 2014. Recuperado de: http://perio.unlp.edu.ar/ojs/index.php/question/ article/viewFile/2073/1877.

Massoni, S. (2013a). Metodologías de la comunicación estratégica del inventario al encuentro sociocultural. Rosario, Argentina. Homo Sapiens Ediciones.

Massoni, S. (2013b). “Comunicación estratégica: matrices de datos en la investigación enactiva”. En Revista Latinoamericana de Ciencias de la Comunicación. Año X No 18, ALAIC, enero-junio 2013. Recuperado de: http://rephip.unr.edu.ar/bitstream/handle/2133/5777/ massoni\%20alaic\%2018.pdf?sequence $=7$.

Massoni, S. (2003). Estrategias de comunicación, un modelo de abordaje de la dimensión comunicacional para el desarrollo sostenible entendido como cambio social conversacional (Tesis doctoral). Universidad de Buenos Aires. Facultad de Filosofía y Letras. Buenos Aires, Argentina.

Massoni, S. (2000). "Estrategias de Comunicación: tiempo de investigarnos vivos”. En Revista de Estudios de la Comunicación Social de Universidad de Guadalajara, México, No 37, Comunicación y Sociedad, junio 2000. Recuperado de: https://drive.google.com/ file/d/0B9WXVQWpgFVdQmtTYXFiZ1FYOUk/view.

Massoni, S. y Pérez, R. (2009). Hacia una teoría general de la estrategia: El cambio de paradigma en el comportamiento humano, la sociedad y las instituciones. Barcelona, España. Ediciones Ariel.

Morin, E. (2005). "Espistemologia de la complejidad". En revista del Departamento de Antropología, Geografía e Historia Facultad de Humanidades y Ciencias de la Educación de la Universidad de Jaén. Año X, No 20, Gazeta de Antropología. Recuperado de: http:// www.ugr.es/ pwlac/G20_02Edgar_Morin.html.

Samaniego, K. (2018). Comunicación en la Organización Artesanal "Los Macaneros" y su incidencia en las políticas públicas de patrimonio cultural (Tesis Doctoral). Universidad Nacional de la Plata, Facultad de Periodismo y comunicación. La Plata, Argentina.

Uranga, W. (2016a). La incidencia como camino para la construcción de ciudadanía. Buenos Aires, Argentina. Ediciones Patria Grande.

Uranga, W. (2016 -b-): “Conocer, transformar, comunicar”. Buenos Aires, Argentina. Ed. Patria Grande. 
Abstract: In this article we will perform an exercise of theoretical and methodological approach that allows us to observe the music of the pluricultural people of the province of Morona Santiago of the Republic of Ecuador as a communication strategy for the resistance of the mining invasion planned by the national state through transnational companies. For this purpose, an exploration will be carried out, from the communication, by the social event called "Upano Fest", an event that takes place year after year in the city of Macas with the intention of observing how, from music, youth is united through to face this intervention.

Keywords: Music - Communication strategy - Resistance - Mining invasion - Amazonia.

Resumo: No presente artigo, vamos realizar um exercício de abordagem teórica e metodológico que permite observar a música dos povos pluriculturais da província de Morona Santiago da República do Equador como estratégia de comunicação para a resistência da invasão mineira planejado pelo Estado nacional através de empresas depois de nacionais. Para isso, será realizada uma exploração, a partir da comunicação, para o evento social chamado "Upano Fest" evento que ocorre ano após ano na cidade de Macas com a intenção observar como, da música, a juventude é unida para enfrentar esta intervenção.

Palavras chave: Música - Estratégia de comunicação - Resistência - Invasão mineradora - Amazônia.

[Las traducciones de los abstracts fueron supervisadas por el autor de cada artículo] 